\title{
BJOG-20-1640.R2 Antenatal testing for sexually transmitted infections: Is this the decade of change?
}

\author{
R Matthew Chico ${ }^{1}$ \\ ${ }^{1}$ London School of Hygiene and Tropical Medicine
}

January 30, 2021

Mini-commentary on BJOG-20-1640.R2: Aetiologic testing compared with syndromic management for sexually transmitted infections in Human Immunodeficiency Virus-infected pregnant women in South Africa: a non-randomised prospective cohort study

Antenatal testing for sexually transmitted infections:

Is this the decade of change?

R. Matthew Chico

Department of Disease Control

Faculty of Infectious \& Tropical Diseases

London School of Hygiene \& Tropical Medicine

Keppel Street, London WC1E 7HT, United Kingdom

The first syndrome-based algorithms were developed in the late 1980s to diagnose and treat Chlamydia trachomatis and Neisseria gonorrhoeae cervicitis in pregnancy (Braddick MR et al. Genitourin Med. 1990;66(2):62-5). These contributed to the first syndromic management guidelines of sexually transmitted infections (STIs) recommended by the World Health Organization (WHO) in 1991 (2001; WHO/HIV_AIDS/2001.01). These guidelines have made important contributions to reducing the burden of curable STIs, particularly in low-resource settings where laboratory facilities and trained staff are lacking. Point of care (POC) tests have since been introduced for syphilis in the antenatal care setting to facilitate rapid treatment (Mabey DC et al. PLoS medicine. 2012;9(6):e1001233). Similar advances in POC testing for other curable STIs have been lacking.

Within this context, Peters et al. (BJOG $2020 \mathrm{xxxx}$ ) conducted an important non-randomised cohort study of HIV-infected pregnant women in South Africa to evaluate POC screening and treatment for C. trachomatis, $N$. gonorrhoeae, and Trichomonas vaginalis $(\mathrm{n}=427 ; 51 \%)$ compared to syndromic management $(\mathrm{n}=414$; 49\%). Among women in the POC screening group, $40.3 \%$ (95\% CI: 35.6, 45.1) were positive for at least one STI, $29.5 \%$ (95\% CI: $25.2,34.0$ ) for C. trachomatis , $5.6 \%$ (95\% CI: 3.6, 8.3) for $N$. gonorrhoeae, and $20.1 \%$ (95\% CI: 95\% CI: 16.4, 24.3) for T. vaginalis . Postpartum, when all women were aetiologically tested, $39 \%$ fewer women had a curable STI who had been POC screened and treated at enrolment compared to women who were provided syndromic management (aRR 0.61; 95\% CI: 0.35-1.05). Although the upper bound of the 95\% confidence interval crossed the null, the intervention effect seems evident: POC testing and treatment reduced the burden of STIs in pregnancy relative to syndromic management in this setting.

The study was underpowered to detect differences in the incidence of preterm birth (23\% vs $23 \%$; aRR 1.2; $95 \%$ CI $0.81-1.8$ ) and low birthweight (15\% vs 13\%; aRR 1.1; 95\% CI: 0.66-1.7). Effect modification 
may have contributed to diluting observable differences. One-fifth of women in the syndromic management group were on antiretroviral therapy at the time of enrolment $(\mathrm{n}=80 ; 19 \%)$ compared to less than one percent in the POC screening and treatment group $(\mathrm{n}=4 ; 0.9 \%)$. Another effect modifier may have been use of cotrimoxazole prophylaxis against opportunistic infections. Overall use was $4.8 \%(\mathrm{n}=40)$, but proportions were not available by treatment group. If antiretroviral use is an indication, women in the syndromic management group may have disproportionately received cotrimoxazole. While cotrimoxazole was unlikely curative of STIs, it is one-part trimethoprim and five-parts sulfamethoxazole, the latter being a sulfanomide. Another sulfanomide combination, sulfadoxine-pyrimethamine, used to prevent the consequences of malaria in pregnancy, has been shown to reduce adverse birth outcomes among pregnant women with curable STIs (Chico RM et al. Clin Infect Dis. 2017 Apr 15; 64(8): 1043-1051), and a recent mediation analysis has demonstrated strong 'non-malarial' protective effects (Roh M et al. The Lancet Global Health. 2020;8(7):e942-e53). Regardless of these possible effect modifiers, Peters et al. provide key evidence in support of randomised controlled trials that compare POC screening and treatment versus syndromic management powered to improved birth outcomes in a range of antenatal care settings. Is this the decade of change?

No disclosures: A completed disclosure of interest form is available to view online as supporting information. 